\title{
Tri-Stimuli-Responsive Biodegradable Theranostics for Mild Hyperthermia Enhanced Chemotherapy
}

Nan Lu, ${ }^{1,4}$ Peng Huang, ${ }^{*}, 2$ Wenpei Fan, ${ }^{4}$ Zhantong Wang, ${ }^{4}$ Yijing Liu, ${ }^{4}$ Sheng Wang, ${ }^{2,4}$ Guofeng Zhang, ${ }^{5}$ Junkai Hu, ${ }^{6}$ Wenfei Liu, ${ }^{1}$ Gang Niu, ${ }^{4}$ Richard D. Leapman, ${ }^{5}$ Guangming Lu, ${ }^{* 1,3}$ and Xiaoyuan Chen ${ }^{*, 4}$

${ }^{1}$ Department of Medical Imaging, Jinling Hospital, Medical School of Nanjing University, Nanjing, 210002 Jiangsu, P.R. China

${ }^{2}$ Guangdong Key Laboratory for Biomedical Measurements and Ultrasound Imaging, School of Biomedical Engineering, Shenzhen University, Shenzhen, 518060 Guangdong, P.R. China State

${ }^{3}$ State Key Laboratory of Analytical Chemistry for Life Science, School of Chemistry and Chemical Engineering, Nanjing University, Nanjing, 210093 Jiangsu, P.R. China ${ }^{4}$ Laboratory of Molecular Imaging and Nanomedicine (LOMIN), National Institute of Biomedical Imaging and Bioengineering (NIBIB), National Institutes of Health, Bethesda, Maryland, 20892, United States

${ }^{5}$ Laboratory of Cellular Imaging and Macromolecular Biophysics, National Institute of Biomedical Imaging and Bioengineering (NIBIB), National Institutes of Health, Bethesda, Maryland, 20892, United States

${ }^{6}$ Department of Chemistry \& Biochemistry, University of Maryland, College Park, Maryland, 20742, United States

\footnotetext{
* Corresponding author. Tel.: +86 258086 0185; Fax: +86 2584804659.

E-mail address: P. Huang (peng.huang@szu.edu.cn); G. Lu (cjr.luguangming@vip.163.com); X. Chen (shawn.chen@nih.gov)
} 


\begin{abstract}
The combination of hyperthermia and chemotherapy is able to greatly enhance the treatment efficacy mainly due to the synergistic interactions between these two treatments. In this study, we propose a new concept of mild hyperthermia enhanced chemotherapy to explore and validate the synergistic mechanism in vitro and in vivo. To do this, a novel kind of biodegradable nanotheranostics based on copper sulfide doped periodic mesoporous organosilica nanoparticles (CuS@PMOs) was constructed via an in situ growth method for light-triggered mild hyperthermia and drug delivery. The as-prepared CuS@PMOs exhibit a high doxorubicin (DOX) loading capacity of $470 \mathrm{mg} / \mathrm{g}$. The DOX release from CuS@PMOs can be precisely controlled by three stimuli, including intracellular glutathione (GSH), acidic environment in tumor cells, and external laser irradiation. Most intriguingly, mild hyperthermia induced by laser-irradiated $\mathrm{CuS}$ nanoparticles can dramatically improve the cell uptake of nanotheranostics both in vitro and in vivo, thus significantly enhancing the chemotherapeutic efficacy for complete tumor growth suppression without recurrence. Meanwhile, the fluorescence recovery following the DOX release can be used as an indicator to monitor the chemotherapeutic progress.
\end{abstract}

Keywords: tri-stimuli-responsive, theranostics, mild hyperthermia, enhanced chemotherapy, CuS, periodic mesoporous organosilicas 


\section{Introduction}

Chemotherapy, an effective and classic cancer treatment tool, has played an extremely important role in the clinic, but it usually causes systemic toxicity because of the off-target phagocytic uptake and nonspecific biodistribution of anticancer drugs [1-3]. In recent years, the combination of chemotherapy and phototherapy (chemophototherapy) is an emerging modality that is attracting interest for solid tumor treatment, holding great potential to enhance the treatment efficacy and reduce the adverse effects [4-8]. However, the nonspecific hyperthermia also improves the drug uptake of normal tissues, resulting in undesirable side effects and toxicity $[9,10]$. To overcome this issue, light-induced hyperthermia with spatiotemporal selectivity has been used in combination with chemotherapy to provide tumor-specific enhanced drug delivery. So far, several photo-activatable nanoplatforms, such as gold nanoshells [11], FeCo/graphitic carbon shell (FeCo/GC) nanocrystals [4], single-walled carbon nanohorns (SWNHs) [12], and graphene-isolated-Au-nanocrystals [13] have been explored as carriers for drug delivery, and simultaneously as photothermal agents for hyperthermia. However, these nanoplatforms have the following limitations: (i) relatively low drug loading capacity due to the limited surface areas of nanoplatforms; (ii) uncontrollable drug release during their circulation; (iii) non-biodegradability that causes potential long-term toxicity; (iv) direct ablation of tumor cells at a temperature higher than $45^{\circ} \mathrm{C}$ or use of high power laser irradiation that inevitably causes adverse damage to normal tissues. Therefore, it is highly desirable to develop a photo-activatable nanoplatform featured with a high drug loading capacity, stimuli-responsive drug release, and good biodegradability for mild hyperthermia enhanced chemotherapy.

Periodic mesoporous organosilica nanoparticles (PMOs), as a new kind of mesoporous silica nanoparticles (MSNs) with organic group-incorporated frameworks, well-defined mesopores, 
large hollow cavity, large surface areas, good biocompatibility and biodegradability, have attracted great attention in nanomedicine, especially in drug delivery with high drug loading capacity [14-16]. Copper sulfide (CuS) nanoparticles have been widely employed as photothermal conversion agents owing to their good light-to-heat conversion property [17-22]. The PMOs contain ample thiol bonds, which can provide nucleating sites for the growth of $\mathrm{CuS}$ nanoparticles. The intermarriage of PMOs and CuS will generate a novel nanoplatform with high drug loading capacity, stimuli-responsive drug release, and good biodegradability for cancer treatment. Herein, we report the design of copper sulfide doped periodic mesoporous organosilica nanoparticles (CuS@PMOs) by in situ growth of ultrasmall CuS nanoparticles in the mesopores of PMOs for photothermally enhanced chemotherapy (Figure 1). The assynthesized CuS@PMOs with a high doxorubicin (DOX) loading capacity demonstrate multistimuli (glutathione (GSH), $\mathrm{pH}$, and laser irradiation)-responsive DOX release.

It is known that mild hyperthermia at $39 \sim 42{ }^{\circ} \mathrm{C}$ induced by low power laser irradiation can enhance the chemotherapy efficacy and avoid damage to noncancerous regions [23, 24]. The temperature rise, though insufficient to kill tumor cells, can increase the cellular sensitivity and vascular permeability, thus leading to enhanced tumor uptake for improved chemotherapeutic effects $[25,26]$. In our case, upon laser irradiation, the moderate hyperthermia generated by CuS is helpful to enhance the cancer cell uptake of DOX-CuS@PMOs both in vitro and in vivo. Furthermore, the intrinsic fluorescence of DOX enables noninvasive monitoring of drug uptake, delivery, and release. Our results demonstrate the excellent role of CuS@PMOs as biodegradable tri-stimuli-responsive cancer theranostics with significant clinical values. 


\section{Materials and Methods}

\subsection{Materials}

Tetraethoxysilane (TEOS), 1,4-Bis(triethoxysily)propane tetrasulfide (TESPTS), cetyltrimethylammonium bromide (CTAB), anhydrous ethanol, concentrated ammonia aqueous solution $\left(\mathrm{NH}_{3} \cdot \mathrm{H}_{2} \mathrm{O}, 25 \mathrm{wt} \%\right)$, concentrated $\mathrm{HCl}$ (37\%), (3-mercaptopropyl)trimethoxysilane, copper chloride $\left(\mathrm{CuCl}_{2}\right)$, sodium sulfide $\left(\mathrm{Na}_{2} \mathrm{~S}\right)$, glutathione $(\mathrm{GSH})$, and DOX in the form of hydrochloride salt were obtained from Sigma-Aldrich (St. Louis, MO, USA). Deionized water (Millipore) with a resistivity of $18 \mathrm{M} \Omega \mathrm{cm}$ was used in all experiments. Minimum Essential Medium (MEM), heat-inactivated fetal bovine serum (FBS), dimethyl sulfoxide (DMSO), $0.05 \%$ trypsin-EDTA, and penicillin-streptomycin solution were obtained from Gibco Laboratories (NY, USA). 3-(4,5-Dimethylthiazol-2-yl)-2,5-diphenyltetrazolium bromide (MTT) was purchased from Thermo Fisher Scientific Inc. (Madison, WI, USA). The U87MG human glioblastoma cell line was bought from American Type Culture Collection (ATCC).

\subsection{Synthesis of hollow yolk-shell structured PMOs.}

Thioether-bridged yolk-shell nanostructured PMOs were synthesized via a surfactant-directed sol-gel method [27]. Briefly, CTAB (0.12 g) was dissolved in a mixed solution containing $\mathrm{NH}_{3} \cdot \mathrm{H}_{2} \mathrm{O}(1 \mathrm{~mL}, 25 \mathrm{wt} \%)$, ethanol $(30 \mathrm{~mL})$, and water $(75 \mathrm{~mL})$. After the solution was heated to $35{ }^{\circ} \mathrm{C}$, a mixture of TEOS $(0.25 \mathrm{~mL})$ and TESPTS $(0.1 \mathrm{~mL})$ was rapidly added into the solution under stirring. The molar ratio of TEOS: TESPTS: CTAB: $\mathrm{NH}_{3} \cdot \mathrm{H}_{2} \mathrm{O}: \mathrm{C}_{2} \mathrm{H}_{5} \mathrm{OH}: \mathrm{H}_{2} \mathrm{O}$ is 1.00: 0.179: 0.294: 11.82: 460: 3720. The above mixture was stirred for $24 \mathrm{~h}$ at $35{ }^{\circ} \mathrm{C}$, and then the white product was collected by centrifugation and washed with ethanol for several times. To obtain yolk-shell structured PMOs, the as-prepared product was dispersed in $30 \mathrm{~mL}$ of water and transferred to a Teflon-lined stainless-steel autoclave. Afterwards, the autoclave was heated up in 
an air flow electric oven at $150{ }^{\circ} \mathrm{C}$ for $24 \mathrm{~h}$, and then cooled down to room temperature. Finally, the as-prepared PMOs were dispersed in $120 \mathrm{~mL}$ of ethanol containing $240 \mu \mathrm{L}$ of $\mathrm{HCl}(37 \%)$ and heated at $60{ }^{\circ} \mathrm{C}$ for $3 \mathrm{~h}$. Then the product was washed with ethanol and water for several times to remove the CTAB molecules. The obtained purified PMOs were redispersed into water for further use.

\subsection{Preparation of $C u S @ P M O s$.}

First, $15 \mathrm{mg}$ of as-prepared hollow yolk-shell structured PMOs was dispersed in $40 \mathrm{~mL}$ of ethanol. After $0.15 \mathrm{~mL}$ of (3-mercaptopropyl)trimethoxysilane and $0.2 \mathrm{~mL}$ of $25 \mathrm{wt} \%$ ammonia aqueous solution were added, the mixture was stirred overnight. PMOs-SH was obtained by centrifugation and washed with ethanol for several times. 5mg of PMOs-SH was dispersed in 10 $\mathrm{mL}$ of $25 \mathrm{mM} \mathrm{CuCl}_{2}$, and the solution was stirred at room temperature overnight. PMOs-Cu ${ }^{2+}$ was obtained and redispersed in $1 \mathrm{~mL}$ of ultrapure water with mild sonication. Afterwards, $5 \mathrm{~mL}$ of $25 \mathrm{mM} \mathrm{Na}_{2} \mathrm{~S}$ aqueous solution was added under stirring at $80{ }^{\circ} \mathrm{C}$ for $15 \mathrm{~min}$, and then the system was transferred into an ice bath to stop the reaction. The products were purified by centrifugation and washed with water for several times.

\subsection{Loading Anti-Cancer Drug.}

Typically, $10 \mathrm{mg}$ of CuS@PMOs was dispersed in $10 \mathrm{~mL}$ of $0.5 \mathrm{mg} / \mathrm{mL}$ DOX phosphatebuffered saline (PBS) solution. After stirring for $24 \mathrm{~h}$ in dark, DOX loaded CuS@PMOs (denoted as DOX-CuS@PMOs) was collected by centrifugation at $11000 \mathrm{rpm}$ for $10 \mathrm{~min}$. Then DOX-CuS@PMOs were further washed with PBS three times, and the washing solutions were collected to measure the unloaded DOX. For cell experiments, CuS@PMOs was loaded with different amounts of DOX so that the concentration of CuS@PMOs was $100 \mu \mathrm{g} / \mathrm{mL}$ at each DOX concentration. 


\subsection{Characterization.}

The particle size and morphology of PMOs and CuS@PMOs were observed and measured by transmission electron microscopy (TEM) on a Tecnai T12 microscope (FEI Company, Hillsboro, OR, USA). Energy-dispersive X-ray (EDX) and high-angle annular dark-field scanning TEM (HAADF-STEM) analyses were performed by using a JEM-2100F transmission electron microscope with an EDX detector system (JEOL USA, Inc., Peabody, MA, USA). The ultraviolet-visible-near infrared (UV-vis-NIR) absorption spectra were obtained by using a Genesys 10S UV-vis spectrophotometer (Thermo Fisher Scientific Inc., Madison, WI, USA). The zeta potential and hydrodynamic size were measured on HORIBA SZ-100 nanoparticle analyzer (HORIBA Instruments Inc., New York, NY, USA). Fourier transform infrared (FT-IR) spectra were recorded on a Nicolet NEXUS870 spectrometer (Nicolet Instruments Inc., Madison, WI, USA).

\subsection{Photothermal Effect Measurement.}

Different concentrations of CuS@PMOs in PBS were prepared, and a total volume of $200 \mu \mathrm{L}$ of each concentration was irradiated by an $808 \mathrm{~nm}$ NIR laser for 3 min with different laser powers. The laser densities were measured by a laser energy meter (Coherent Inc., Santa Clara, CA, USA). The temperature changes of the solution were recorded by using an infrared (IR) thermal imaging system.

2.7. Calculation of Mass Absorption Coefficient and Photothermal Conversion Efficiency at 808 $n m$.

Mass absorption coefficient of CuS@PMOs is calculated by the following formula.

$$
a=\frac{A}{b c}
$$

where $A$ is the absorbance of CuS@PMOs at $808 \mathrm{~nm}, b$ is the length of quartz cell, and $c$ is the concentration of CuS@PMOs. 
The photothermal conversion efficiency of CuS@PMOs is calculated by the following equation.

$$
\eta=\frac{h S\left(T_{\max }-T_{a m b}\right)-Q_{0}}{I\left(1-10^{-A}\right)}
$$

where $h$ is heat transfer coefficient, $S$ stands for the surface area, $T_{\text {max }}$ is equilibrium temperature, $T_{a m b}$ is surrounding ambient temperature, $Q_{0}$ is heat absorption of quartz cell, $I$ express the laser power, and $A$ is the absorbance of CuS@PMOs at 808 nm.

If the heat input is equal to the heat output,

$$
h S=\frac{\sum_{i} m_{i} C_{p, i}}{\tau_{s}} \approx \frac{m_{\mathrm{H}_{2} \mathrm{O}} C_{\mathrm{H}_{2} \mathrm{O}}}{\tau_{s}}
$$

where $m_{\mathrm{H}_{2} \mathrm{O}}$ express the weight of water, $C_{\mathrm{H}_{2} \mathrm{O}}$ is the specific heat capacity of water, and $\tau_{S}$ stands for time constant of the sample.

At cooling stage,

$$
t=-\tau_{s} \ln \theta=-\tau_{s} \ln \frac{T-T_{a m b}}{T_{\max }-T_{a m b}}
$$

Therefore, $\tau_{s}$ can be calculated by using the linear regression curve between cooling stage and negative natural logarithm of driving force temperature of CuS@PMOs.

\subsection{In Vitro GSH, pH and Laser-Responsive Drug Release.}

In vitro GSH and $\mathrm{pH}$-responsive drug release was executed in PBS solution at different GSH concentrations of 0,5 and $10 \mathrm{mM}$ and different $\mathrm{pH}$ of 7.4 and 5.5. Briefly, the above-prepared DOX-CuS@PMOs (1.5 mg) were dispersed in PBS $(1.5 \mathrm{~mL})$ at different GSH concentrations $(0$, 5 and $10 \mathrm{mM}$ ) and different $\mathrm{pH}(7.4$ and 5.5), which was further shaken with a speed of $100 \mathrm{rpm}$ at $37^{\circ} \mathrm{C}$. For each cycle of laser triggered drug release, $1.5 \mathrm{~mL}$ of PBS solution at different GSH concentrations (0 and $5 \mathrm{mM})$ and different pH (7.4 and 5.5) containing DOX-CuS@PMOs (1 $\mathrm{mg} / \mathrm{mL}$ ) was directly irradiated by $808 \mathrm{~nm}$ NIR laser for 2 min. Afterwards, the solution was put in a shaking table at $100 \mathrm{rpm}$ and $37{ }^{\circ} \mathrm{C}$ for $1 \mathrm{~h}$. The power density of laser was $0.5 \mathrm{~W} / \mathrm{cm}^{2}$. At certain time intervals, each drug releasing system was centrifuged and redispersed in $1.5 \mathrm{~mL}$ of 
fresh media. The obtained clear supernatant was used to determine the released drug amount by a UV-vis spectrometer at a wavelength of $490 \mathrm{~nm}$.

\subsection{In Vitro Cytotoxicity Assay.}

U87MG human glioblastoma cells were cultured in MEM supplemented with 10\% FBS in a humidified atmosphere with $5 \% \mathrm{CO}_{2}$ at $37{ }^{\circ} \mathrm{C}$. The cytotoxicity of CuS@PMOs or DOXCuS@PMOs was measured on U87MG cells by MTT assay. The cells were cultured in 96-well plates at a density of $1 \times 10^{4}$ cells per well and incubated for $24 \mathrm{~h}$. Then the medium was replaced with $100 \mu \mathrm{L}$ of fresh MEM containing CuS@PMOs, free DOX, or DOX-CuS@PMOs at different concentrations. For laser irradiation group, cells were immediately irradiated by 808 $\mathrm{nm}$ laser of $0.8 \mathrm{~W} / \mathrm{cm}^{2}$ for $30 \mathrm{~min}$, the power of laser density might be tuned to maintain the temperature at $41 \sim 42{ }^{\circ} \mathrm{C}$ monitored by using IR thermal imaging system. Afterwards, the medium was replaced and incubated for another $24 \mathrm{~h}$. Then $10 \mu \mathrm{L}$ of MTT $(5 \mathrm{mg} / \mathrm{mL}$ in PBS, pH 7.4) was added to each well and the plate was incubated at $37^{\circ} \mathrm{C}$ for another $4 \mathrm{~h}$. After the medium was removed, the intracellular formazan crystals in cells were dissolved into $150 \mu \mathrm{L}$ of DMSO. Finally, the absorbance was measured by a microplate reader at $570 \mathrm{~nm}$ (BioTek Instruments, Winooski, VT, USA).

\subsection{In Vivo Cancer Treatment.}

All animal experiments were performed following a National Institutes of Health Animal Care and Use Committee (NIHACUC) approved protocol. Female athymic nude mice with tumors on the right flank were prepared by subcutaneously injection of $2 \times 10^{6}$ U87MG cells. The mice were used for chemotherapy when the tumors reached about $70 \mathrm{~mm}^{3}$. The tumor-bearing mice were divided into five groups with 5-6 mice in each group (DOX-CuS@PMOs with laser treatment, DOX-CuS@PMOs only, free DOX,CuS@PMOs with laser treatment, and PBS only). 
$100 \mu \mathrm{L}$ of particle PBS solution or PBS was intratumorally injected, and equivalent DOX dose was $3 \mathrm{mg} / \mathrm{kg}$. Mice in laser irradiation group were exposed to $808 \mathrm{~nm}$ laser of $0.3 \mathrm{~W} / \mathrm{cm}^{2}$ for 30 min, and the temperature was controlled at $41 \sim 42{ }^{\circ} \mathrm{C}$ by tuning the laser power density. The same administration was repeated at the $3^{\text {rd }}$ and $5^{\text {th }}$ day after treatment. Tumor volumes and body weight were measured every 2 days after the treatment. Tumor volumes were calculated by the formula: $\mathrm{V}=$ width $^{2} \times$ length $/ 2$. The relative tumor volume and body weight was normalized to their initial tumor volume and body weight respectively.

\subsection{Histological Staining.}

Tumors from the five groups were collected at 18 days after treatment. Tissues were fixed in $10 \%$ formalin solution and stored at room temperature. Hematoxylin and eosin $(\mathrm{H} \& \mathrm{E})$ staining sections (Histoserv, Germantown, MD, USA) were examined by a BX41 bright field microscopy (Olympus, Tokyo, Japan).

\section{Results and Discussion}

PMOs with thioether-bridged yolk-shell structure were synthesized according to our previous report [27]. CuS nanoparticles were in situ synthesized in the mesopores of PMOs through the binding force between $\mathrm{Cu}^{2+}$ and thiol group. Transmission electron microscopy (TEM) images shown in Figure 2a \& b exhibit a uniform yolk-shell structure of PMOs with a diameter of around $250 \mathrm{~nm}$. Accompanied with the formation of $\mathrm{CuS}$ nanoparticles, the color changed from white (inset of Figure 2a) to dark green (inset of Figure 2b). As shown in Figure 2c, the ultraviolet-visible-near infrared (UV-vis-NIR) absorbance spectra indicate that CuS@PMOs display strong absorbance in the NIR region, while PMOs show virtually no absorbance in that region. The high-angle annular dark-field scanning TEM (HAADF-STEM) and energy- 
dispersive X-ray (EDX) element mapping uncover that CuS@PMOs exhibit a uniform distribution of $\mathrm{Si}, \mathrm{O}, \mathrm{S}$, and $\mathrm{Cu}$ elements (Figure 2d). All these results suggest successful in situ growth of $\mathrm{CuS}$ in the pores of PMOs. The content of $\mathrm{CuS}$ in $\mathrm{CuS} @ \mathrm{PMOs}$ was further measured by using inductively coupled plasma (ICP) analysis, indicating $100 \mu \mathrm{g}$ of copper per $\mathrm{mg}$ of CuS@PMOs. The zeta potential of PMOs is negative, around $-26.8 \mathrm{mV}$, but turned into $58.0 \mathrm{mV}$ after the formation of $\mathrm{CuS}$ (Figure 2e), which is consistent with other studies [28]. The large surface area of $658 \mathrm{~m}^{2} / \mathrm{g}$ (Figure S1a) and uniform pore size of $2.67 \mathrm{~nm}$ (Figure S1b) make CuS@PMOs suitable for drug delivery. After the loading DOX into the cavity of PMOs, the zeta potential of DOX-CuS@PMOs changed to $74.2 \mathrm{mV}$, higher than that of CuS@PMOs (Figure 2e). CuS@PMOs and DOX-CuS@PMOs display narrow size distribution with average hydrodynamic diameters of 262 and $299 \mathrm{~nm}$, respectively (Figure 2f), showing their high dispersity in aqueous solution. Moreover, the size distributions of CuS@PMOs in PBS (Figure S2a), FBS (Figure S2b), and MEM (Figure S2c) indicate good stability in different conditions. As shown in the Fourier transform infrared (FT-IR) spectra (Figure 2g), CuS@PMOs demonstrate the characteristic bands of C-H bond at 2930/1450/1410 $\mathrm{cm}^{-1}$, C-S bond at 694 $\mathrm{cm}^{-1}$, and $-\mathrm{SH}$ bond at $580 \mathrm{~cm}^{-1}[14,29]$. After DOX was loaded into the hollow PMOs through electrostatic interaction, there appeared a new band at $1700 \mathrm{~cm}^{-1}$, which was assigned to $\mathrm{C}=\mathrm{O}$ carbonyl stretching of DOX [30]. We further measured the drug loading capacity of CuS@ PMOs by using DOX as a model drug. About $470 \mathrm{mg}$ of DOX can be loaded in 1g of CuS@ PMOs with a high drug loading efficiency of $94 \%$.

Inspired by the strong NIR absorbance of CuS@PMOs, the corresponding light-to-heat conversion efficiency was investigated in two different ways: different concentrations of CuS@PMOs (50 200 $\mu \mathrm{g} / \mathrm{mL})$ were exposed to $808 \mathrm{~nm}$ laser irradiation with power density of 
$0.8 \mathrm{~W} / \mathrm{cm}^{2}$ for $3 \mathrm{~min}$ (Figure S3a), and $100 \mu \mathrm{g} / \mathrm{mL}$ of CuS@PMOs were exposed to $808 \mathrm{~nm}$ laser irradiation with different power densities ranging from 0.5 to $1.2 \mathrm{~W} / \mathrm{cm}^{2}$ (Figure $\mathrm{S} 3 \mathrm{~b}$ ). The corresponding photothermal images of different solutions were shown in Figure S4. Obviously, both concentration-dependent and laser power density-dependent temperature increases for CuS@PMOs aqueous solutions were observed, which enabled the precise control of temperature below $42{ }^{\circ} \mathrm{C}$ for mild hyperthermia by adjusting the laser power density or $\mathrm{CuS} @ \mathrm{PMOs}$ concentration. Meanwhile, CuS@PMOs show an excellent yet stable light-to-heat conversion property within five cycles of laser irradiation (Figure S3c). The mass absorption coefficient of CuS@PMOs is calculated to be $2.2 \mathrm{~L} /(\mathrm{g} \cdot \mathrm{cm})$, and $\tau_{s}$ can be determined by using the linear regression curve between cooling stage and negative natural logarithm of driving force temperature of CuS@PMOs (Figure S5). As a result, the photothermal conversion efficiency of CuS@PMOs is calculated to be $62.5 \%$.

Disulfide bonds physiologically respond to the intracellular GSH, which has been widely applied in smart drug delivery systems [15, 31-33]. Since the concentration of intracellular GSH (1-11 $\mathrm{mM})$ for dissociating the disulfide bonds within the framework of PMOs is much higher than that of extracellular $(10 \mu \mathrm{M})$ in cancer cells, the payload release can be triggered in vitro $[34,35]$. The dissociation of CuS@PMOs shown in Figure S6 suggests that CuS@PMOs can be degraded in GSH solution. As shown in Figure 3a, GSH-triggered DOX release from DOXCuS@PMOs was performed in neutral phosphate-buffered saline $(\mathrm{PBS})$ solution $(\mathrm{pH}=7.4)$ at different GSH concentrations of 0,5 , or $10 \mathrm{mM}$. The DOX release rate is strongly dependent on the GSH concentration. 20\% of DOX is released within first $1 \mathrm{~h}$ in $10 \mathrm{mM} \mathrm{GSH}$ solution, and the release percentage keeps increasing in the following $7 \mathrm{~h}$, reaching more than $40 \%$ within $8 \mathrm{~h}$. In contrast, without GSH, the release of DOX was only $13 \%$ in $8 \mathrm{~h}$. Drug release profiles were 
further investigated in acidic PBS solutions with various GSH concentrations, simulating intracellular endo/lysosomal ( $\mathrm{pH} 5.5$ ) environment (Figure 3b). In acidic environment, the rapid DOX release is observed, reaching $62.5 \%$ at the end of $8 \mathrm{~h}$ in $10 \mathrm{mM}$ GSH solution, much higher than that in neutral solution. After validating that the DOX release from DOX-CuS@PMOs could be triggered by both GSH and $\mathrm{pH}$, we further examined the effect of laser irradiation on the DOX release. As shown in Figure 3c, laser irradiation can promote drug release to some extent at $\mathrm{pH}=7.4$. However, $\mathrm{DOX}$ release is dramatically accelerated upon laser irradiation in acidic environment, especially in the presence of GSH (Figure 3d). After four cycles of laser irradiation, the release percentage of DOX reaches around $60 \%$ at GSH concentration of $5 \mathrm{mM}$ in acidic environment, which is much higher than that of $27 \%$ at the same GSH concentration in neutral condition (Figure 3c), also higher than that of $33.6 \%$ at the same time point in the same environment but without laser irradiation (Figure 3b). The light-triggered DOX release enhancement from DOX-CuS@PMOs is mainly attributed to the dissociation of DOX from PMOs caused by the heat generated by CuS@PMOs upon laser irradiation [36]. After DOX was released from DOX-CuS@PMOs under various situations, zeta potentials of DOX-CuS@PMOs were decreased (Figure S7), which imply the dissociation of DOX-CuS@PMOs.

Considering the high GSH concentration and acidic environment in cancer cells, DOX is firstly released from the nanotheranostics once DOX-CuS@PMOs enter the cells. Further drug release is triggered by external laser irradiation. As a result, the triple stimuli enable sequential, more efficient and complete drug release from drug carriers than mono or dual stimuli, and in the meantime avoid premature extracellular release during the circulation, thus reducing side effects to normal tissues. Taking into account that laser irradiation time and power density can be 
controlled accurately, the GSH/pH/laser tri-stimuli-responsive DOX-CuS@PMOs are expected to be applied to "on demand" chemotherapy of cancer.

Encouraged by the light-to-heat conversion property of $\mathrm{CuS}$, mild hyperthermia enhanced cellular uptake of DOX-CuS@PMOs was firstly investigated on U87MG human glioblastoma cells, which were divided into four groups: control, free DOX, DOX-CuS@PMOs, and DOXCuS@PMOs with laser irradiation for $30 \mathrm{~min}$. After $30 \mathrm{~min}$ incubation, U87MG cells were fixed and stained with DAPI (4',6-diamidino-2-phenylindole) to visualize the blue fluorescence of nuclei, which was easily differentiated from the red fluorescence of DOX. As seen from the confocal images of U87MG cells subjected to different conditions (Figure 4a), no red fluorescence signal was observed for the cells in the control group. For the cells incubated with DOX-CuS@PMOs without laser irradiation, DOX was released in response to intracellular GSH and acidic environment, resulting in only sporadic red fluorescence signal shown in cytoplasm. In comparison, the DOX signal was much stronger both in cytoplasm and nuclei after adding laser irradiation, because mild hyperthermia induced by laser not only enhanced the cell uptake of DOX-CuS@PMOs, but also greatly stimulated the DOX release. To further confirm this observation, flow cytometry was used to quantify the red fluorescence signal intensity of DOX. Figure $4 b, 4 c$ and Figure S8 show the fluorescence intensities of cells treated with DOXCuS@PMOs and laser irradiation was much stronger than that without laser exposure at all tested DOX concentrations. These results were also evidenced by ICP analysis. In laser irradiation group, the uptake of $\mathrm{Cu}$ was as high as nearly 60-fold of that of the control group, and 2-fold of that in DOX-CuS@PMOs without laser irradiation (Figure 4d).

In this study, we also provided the cell TEM images to directly and obviously demonstrate the enhanced cell uptake of nanotheranostics induced by mild hyperthermia. As shown in Figure 
$5 \mathrm{~b}$, after $30 \mathrm{~min}$ of incubation, only a small number of nanotheranostics were internalized by cells without laser exposure. In contrast, the cell uptake of CuS@PMOs was enhanced significantly by low power laser irradiation (Figure 5c and 5d) mainly because the local mild hyperthermia induced minor disruptions to cell membrane for improved intracellular delivery of nanotheranostics $[25,26]$.

The above results strongly confirm that mild hyperthermia can significantly enhance the cell uptake of CuS@PMOs. We hypothesize that after DOX is loaded into CuS@PMOs, the enhanced uptake of DOX-CuS@PMOs would remarkably improve the chemotherapeutic effect against tumor cells. The cytotoxicity of CuS@PMOs was tested and shown in Figure 6a. As both $\mathrm{CuS}$ and PMOs are of good biocompatibility [16, 37], CuS@PMOs $(0 \sim 100 \mu \mathrm{g} / \mathrm{mL})$ caused negligible toxicity to U87MG cells. For the mild hyperthermia group, all the cell viabilities are over $90 \%$, which implies that the laser irradiation does not produce cell killing effect. As expected, mild hyperthermia enhanced cellular uptake of DOX-CuS@PMOs and thus induced much higher cell death rate (Figure 6b). Live and dead cells can be differentiated by co-staining with Calcein acetoxymethyl ester (CalceinAM) and propidium iodide (PI) (Figure 6c). In control and DOX-CuS@PMOs groups, almost all cells remained alive. For DOX-CuS@PMOs plus laser irradiation group, most of the cells were dead, corroborating with the results seen in Figure $6 \mathrm{~b}$.

The above in vitro cell experiment results have shown that mild heating can significantly promote the cellular internalization of the nanotheranostics, but do not produce significant photothermal cell killing effect. Therefore, the same experiment parameters (e.g., the temperature, laser irradiation time, etc.) were set for inducing mild hyperthermia in U87MG tumor xenograft model. After injection of DOX-CuS@PMOs, the tumors were exposed to a low power density of $808 \mathrm{~nm}$ laser irradiation for $30 \mathrm{~min}$ to control the temperature at $41 \sim 42{ }^{\circ} \mathrm{C}$. 
Fluorescence imaging was used to monitor the DOX fluorescence signal change of the tumor area. As seen from Figure 7a, immediately after drug administration, the fluorescence signal of free DOX is higher than the other two groups, owing to the quenched DOX fluorescence in DOX-CuS@PMOs. However, DOX signal is gradually decreased in the free DOX group due to the clearance of DOX from circulation and the tumor region. In contrast, the DOX fluorescence signal is gradually recovered over time in the groups treated with DOX-CuS@PMOs because of the DOX release from nanotheranostics in response to intracellular GSH and low $\mathrm{pH}$ once the nanotheranostics are internalized by tumor cells (Figure $7 \mathrm{~b}$ and $7 \mathrm{c}$ ). Moreover, laser irradiation can contribute to the elevated cell uptake of nanotheranostics as well as increased intracellular drug release, which result in the strong time-dependent DOX fluorescence signal increase in the group with laser irradiation, contrary to the limited fluorescence change in the group without laser irradiation. To further confirm the enhanced tumor cell uptake in vivo, bio-TEM images of tumors injected with DOX-CuS@PMOs with or without laser irradiation were compared in Figure 7d, 7e, and S9. A large number of DOX-CuS@PMOs were found in the peri-nucleus region in the laser irradiation group (Figure 7d and S9a). However, without laser irradiation, only few nanotheranostics entered the tumor cells (Figure 7e and S9b). Therefore, it can be concluded that low power density of laser irradiation can greatly enhance the tumor cell uptake of nanotheranostics, and more importantly facilitate their distribution in peri-nucleus region. Thanks to the low $\mathrm{pH}$ and GSH-rich microenvironment, the DOX can be responsively released to easily enter the nucleus for killing the tumor cells.

Finally, the mild hyperthermia enhanced chemotherapeutic effect was evaluated in U87MG tumors. As shown in Figure 8a, during the low power density of laser irradiation for $30 \mathrm{~min}$, the temperature of tumor treated with DOX-CuS@PMOs is maintained at $41 \sim 42{ }^{\circ} \mathrm{C}$, so the 
generated mild hyperthermia is insufficient to shrink tumors [38, 39]. As well, the temperature is not elevated in the control group injected with PBS. Tumor growth rate was inhibited to some degree when treated with DOX-CuS@PMOs only, mainly because the low tumor uptake of nanotheranostics could not maintain the chemotherapeutic effect. However, in the group with DOX-CuS@PMOs plus laser irradiation, both the tumor growth rate and tumor size were significantly suppressed as compared to the other groups (control, CuS@PMOs with laser, free DOX, and DOX-CuS@PMOs without laser) (Figure 8b). In addition, the bodyweight in the group treated with free DOX decreased by $12.75 \%$, indicating the potential toxicity of free DOX, although free DOX have somewhat better treatment efficacy than DOX-CuS@PMOs only. Excitingly, the treatment of DOX-CuS@PMOs plus laser irradiation did not cause noticeable weight loss (Figure 8c), indicating the high bio-safety of DOX-CuS@PMOs. Moreover, the mice treated with DOX-CuS@PMOs plus laser irradiation had a much longer survival period than the other four groups (Figure 8d). Afterwards, hematoxylin and eosin (H\&E) staining was performed to investigate the chemotherapeutic effects and in vivo toxicity of DOX-CuS@PMOs. As shown in Figure 8e, a large area of apoptosis was observed in tumors treated with DOX-CuS@PMOs plus laser irradiation, which is in sharp contrast to only a small area of apoptosis in tumors treated with free DOX and DOX-CuS@PMOs only. In addition, the tumor tissues in the control and CuS@PMOs with laser groups are dense and rich in vasculature, indicating the rapid tumor growth in these two groups. Major organs of mice treated with DOXCuS@PMOs upon laser irradiation were also collected at 18 and 35 days after the treatment. No obvious side effects were found in these tissues (Figure S10), suggesting the negligible long-term adverse toxicity. All the above results demonstrate the promising potential of DOX-CuS@PMOs for mild hyperthermia enhanced cancer chemotherapy in vivo. 


\section{Conclusions}

In summary, we have developed copper sulfide-periodic mesoporous organosilica nanoparticles (CuS@PMOs) for GSH/pH/laser triple stimuli-responsive drug release and mild hyperthermia enhanced chemotherapy, by taking advantages of the low $\mathrm{pH}$ and GSH-rich tumor microenvironment and photothermal effect of $\mathrm{CuS}$. Ultrasmall $\mathrm{CuS}$ nanoparticles were in situ synthesized in the mesopores of PMOs via a straightforward method, and the internal hollow cavity is ideal for loading anti-cancer drug DOX. The as-prepared CuS@PMOs exhibit excellent drug loading ability, good biocompatibility, stability, and photothermal ability. It is for the first time validated both in vitro and in vivo that the enhanced chemotherapeutic effect of DOX$\mathrm{CuS} @ \mathrm{PMOs}$ is attributed to the elevated tumor uptake of nanotheranostics induced by mild hyperthermia (below $42{ }^{\circ} \mathrm{C}$ ). Upon low power density of laser irradiation, DOX-CuS @ PMOs are facilely internalized by tumor cells and accumulated in the peri-nucleus region, where DOX molecules are easy to enter nucleus for dramatically killing cancer cells and suppressing tumor growth. In the meantime, the DOX delivery and release are also monitored by the red fluorescence signal. The proposed concept of mild hyperthermia enhanced chemotherapy and the systemic validation using the triple stimuli-responsive nanotheranostics in this study may possibly be a complex variable treatment, but hold great potential to controllable drug release, enhanced chemotherapy efficacy with low dose of drug administration as well as reduced side effects in future clinical applications.

\section{Acknowledgement}


We greatly appreciate financial support from the National Key Basic Research Program of the PRC (2014CB744504 and 2014CB744501), the Major International (Regional) Joint Research Program of China (81120108013), the National Natural Science Foundation of China (81201175 81530054, 81401465 and 51573096), the Natural Science Foundation of Jiangsu Province (BK20130863), and the National Science Foundation for Post-doctoral Scientists of China (2013T60939 and 2012M521934). We also acknowledge the support from the Intramural Research Program (IRP), National Institute of Biomedical Imaging and Bioengineering (NIBIB), National Institutes of Health (NIH). 


\section{REFERENCES}

[1] P.G. Morris, A.B. Lassman, Medical oncology: Optimizing chemotherapy and radiotherapy for anaplastic glioma, Nat. Rev. Clin. Oncol. 7 (2010) 428-430.

[2] A. Gabizon, M. Bradbury, U. Prabhakar, W. Zamboni, S. Libutti, P. Grodzinski, Cancer nanomedicines: closing the translational gap, Lancet 384 (2014) 2175-2176.

[3] S. Wang, P. Huang, X. Chen, Stimuli-responsive programmed specific targeting in nanomedicine, Acs Nano 10 (2016) 2991-2994.

[4] S.P. Sherlock, S.M. Tabakman, L. Xie, H. Dai, Photothermally enhanced drug delivery by ultrasmall multifunctional FeCo/graphitic shell nanocrystals, Acs Nano 5 (2011) 1505-1512.

[5] Z. Zhang, J. Wang, C. Chen, Near-infrared light-mediated nanoplatforms for cancer thermochemotherapy and optical imaging, Adv. Mater. 25 (2013) 3869-3880.

[6] S. Baek, R.K. Singh, T.H. Kim, J.W. Seo, U.S. Shin, W. Chrzanowski, H.W. Kim, Triple hit with drug carriers: $\mathrm{pH}$ - and temperature-responsive theranostics for multimodal chemo- and photothermal therapy and diagnostic applications, ACS Appl. Mater. Interfaces 8 (2016) 8967-8979.

[7] J. Li, M. Zhou, F. Liu, C. Xiong, W. Wang, Q. Cao, X. Wen, J.D. Robertson, X. Ji, Y.A. Wang, S. Gupta, C. $\mathrm{Li}$, Hepatocellular carcinoma: intra-arterial delivery of doxorubicin-loaded hollow gold nanospheres for photothermal ablation-chemoembolization therapy in rats, Radiology 281 (2016) 427-435.

[8] D. Luo, K.A. Carter, D. Miranda, J.F. Lovell, Chemophototherapy: an emerging treatment option for solid tumors, Adv. Sci. 4 (2017) 1600106

[9] M.H. Falk, R.D. Issels, Hyperthermia in oncology, Int. J. Hyperthermia 17 (2001) 1-18.

[10] Y. Tang, A.J. McGoron, Combined effects of laser-ICG photothermotherapy and doxorubicin chemotherapy on ovarian cancer cells, J. Photochem. Photobiol. B 97 (2009) 138-144.

[11] S.J. Madsen, E.C. Shih, Q. Peng, C. Christie, T. Krasieva, H. Hirschberg, Photothermal enhancement of chemotherapy mediated by gold-silica nanoshell-loaded macrophages: in vitro squamous cell carcinoma study, J. Biomed. Opt. 21 (2016) 18004.

[12] D. Chen, C. Wang, F. Jiang, Z. Liu, C. Shu, L.-J. Wan, In vitro and in vivo photothermally enhanced chemotherapy by single-walled carbon nanohorns as a drug delivery system, J. Mater. Chem. B 2 (2014) 4726-4732.

[13] X. Bian, Z.L. Song, Y. Qian, W. Gao, Z.Q. Cheng, L. Chen, H. Liang, D. Ding, X.K. Nie, Z. Chen, W. Tan, Fabrication of graphene-isolated-Au-nanocrystal nanostructures for multimodal cell imaging and photothermal-enhanced chemotherapy, Sci. Rep. 4 (2014) 6093-6111. 
[14] N. Lu, Y. Tian, W. Tian, P. Huang, Y. Liu, Y. Tang, C. Wang, S. Wang, Y. Su, Y. Zhang, J. Pan, Z. Teng, G. $\mathrm{Lu}$, Smart cancer cell targeting imaging and drug delivery system by systematically engineering periodic mesoporous organosilica nanoparticles, ACS Appl. Mater. Interfaces 8 (2016) 2985-2993.

[15] Y. Chen, Q. Meng, M. Wu, S. Wang, P. Xu, H. Chen, Y. Li, L. Zhang, L. Wang, J. Shi, Hollow mesoporous organosilica nanoparticles: a generic intelligent framework-hybridization approach for biomedicine, J. Am. Chem. Soc. 136 (2014) 16326-16334.

[16] Z. Teng, X. Su, Y. Zheng, J. Zhang, Y. Liu, S. Wang, J. Wu, G. Chen, J. Wang, D. Zhao, G. Lu, A facile multi-interface transformation approach to monodisperse multiple-shelled periodic mesoporous organosilica hollow spheres, J. Am. Chem. Soc. 137 (2015) 7935-7944.

[17] M. Zhou, J. Li, S. Liang, A.K. Sood, D. Liang, C. Li, CuS nanodots with ultrahigh efficient renal clearance for positron emission tomography imaging and image-guided photothermal therapy, Acs Nano 9 (2015) 7085-7096.

[18] F. Chen, H. Hong, S. Goel, S.A. Graves, H. Orbay, E.B. Ehlerding, S. Shi, C.P. Theuer, R.J. Nickles, W. Cai, In vivo tumor vasculature targeting of CuS@MSN based theranostic nanomedicine, Acs Nano 9 (2015) 3926-3934.

[19] S. Goel, F. Chen, W. Cai, Synthesis and biomedical applications of copper sulfide nanoparticles: from sensors to theranostics, Small 10 (2014) 631-645.

[20] R. Liu, L. Jing, D. Peng, Y. Li, J. Tian, Z. Dai, Manganese (II) chelate functionalized copper sulfide nanoparticles for efficient magnetic resonance/photoacoustic dual-modal imaging guided photothermal therapy, Theranostics 5 (2015) 1144-1153.

[21] Z. Zha, S. Zhang, Z. Deng, Y. Li, C. Li, Z. Dai, Enzyme-responsive copper sulphide nanoparticles for combined photoacoustic imaging, tumor-selective chemotherapy and photothermal therapy, Chem. Commun. 49 (2013) 3455-3457.

[22] J. Lin, M. Wang, H. Hu, X. Yang, B. Wen, Z. Wang, O. Jacobson, J. Song, G. Zhang, G. Niu, P. Huang, X. Chen, Multimodal-imaging-guided cancer phototherapy by versatile biomimetic theranostics with UV and gamma-irradiation protection, Adv. Mater. 28 (2016) 3273-3279.

[23] D.K. Chatterjee, P. Diagaradjane, S. Krishnan, Nanoparticle-mediated hyperthermia in cancer therapy, Ther. Deliv. 2 (2011) 1001-1014.

[24] J.P. May, S.D. Li, Hyperthermia-induced drug targeting, Expert Opin. Drug Deliv. 10 (2013) 511-527.

[25] X. Sun, G. Zhang, R.S. Keynton, M.G. O'Toole, D. Patel, A.M. Gobin, Enhanced drug delivery via hyperthermal membrane disruption using targeted gold nanoparticles with PEGylated protein-G as a cofactor, Nanomedicine 9 (2013) 1214-1222.

[26] T.S. Hauck, T.L. Jennings, T. Yatsenko, J.C. Kumaradas, W.C.W. Chan, Enhancing the toxicity of cancer chemotherapeutics with gold nanorod hyperthermia, Adv. Mater. 20 (2008) 3832-3838. 
[27] Z. Teng, X. Su, B. Lee, C. Huang, Y. Liu, S. Wang, J. Wu, P. Xu, J. Sun, D. Shen, W. Li, G. Lu, Yolk-shell structured mesoporous nanoparticles with thioether-bridged organosilica frameworks, Chem Mater 26 (2014) 5980-5987.

[28] D. Fullston, D. Fornasiero, J. Ralston, Zeta potential study of the oxidation of copper sulfide minerals, Colloids Surf., A 146 (1999) 113-121.

[29] I. Cesarino, E.T.G. Cavalheiro, Characterization of thiol-functionalised silica films deposited on electrode surfaces, Mater. Res.-Ibero.-Am. J. 11 (2008) 465-469.

[30] Y.X. Tang, Z.G. Teng, Y. Liu, Y. Tian, J. Sun, S.J. Wang, C.Y. Wang, J.D. Wang, G.M. Lu, Cytochrome C capped mesoporous silica nanocarriers for $\mathrm{pH}$-sensitive and sustained drug release, J. Mater. Chem. B 2 (2014) 4356-4362.

[31] J.P. Lai, B.P. Shah, E. Garfunkel, K.B. Lee, Versatile fluorescence resonance energy transfer-based mesoporous silica nanoparticles for real-time monitoring of drug release, Acs Nano 7 (2013) 2741-2750.

[32] S. Chen, Q. Lei, S.Y. Li, S.Y. Qin, H.Z. Jia, Y.J. Cheng, X.Z. Zhang, Fabrication of dual responsive codelivery system based on three-armed peptides for tumor therapy, Biomaterials 92 (2016) 25-35.

[33] S. Wang, P. Huang, X. Chen, Hierarchical targeting strategy for enhanced tumor tissue accumulation/retention and cellular internalization, Adv. Mater. 28 (2016) 7340-7364.

[34] S. Bauhuber, C. Hozsa, M. Breunig, A. Gopferich, Delivery of nucleic acids via disulfide-based carrier systems, Adv. Mater. 21 (2009) 3286-3306.

[35] M.H. Lee, Z. Yang, C.W. Lim, Y.H. Lee, S. Dongbang, C. Kang, J.S. Kim, Disulfide-cleavage-triggered chemosensors and their biological applications, Chem. Rev. 113 (2013) 5071-5109.

[36] S. Shen, H.Y. Tang, X.T. Zhang, J.F. Ren, Z.Q. Pang, D.G. Wang, H.L. Gao, Y. Qian, X.G. Jiang, W.L. Yang, Targeting mesoporous silica-encapsulated gold nanorods for chemo-photothermal therapy with near-infrared radiation, Biomaterials 34 (2013) 3150-3158.

[37] Z. Wang, P. Huang, O. Jacobson, Y. Liu, L. Lin, J. Lin, N. Lu, H. Zhang, R. Tian, G. Niu, G. Liu, X. Chen, Biomineralization-inspired synthesis of copper sulfide-ferritin nanocages as cancer theranostics, Acs Nano 10 (2016) 3453-3460.

[38] X. Sun, X. Huang, X. Yan, Y. Wang, J. Guo, O. Jacobson, D. Liu, L.P. Szajek, W. Zhu, G. Niu, D.O. Kiesewetter, S. Sun, X. Chen, Chelator-free (64)Cu-integrated gold nanomaterials for positron emission tomography imaging guided photothermal cancer therapy, Acs Nano 8 (2014) 8438-8446.

[39] Y. Lyu, Y. Fang, Q. Miao, X. Zhen, D. Ding, K. Pu, Intraparticle molecular orbital engineering of semiconducting polymer nanoparticles as amplified theranostics for in vivo phtoacoustic imaging and photothermal therapy. Acs Nano 10 (2016) 4472-4481. 


\section{Figure Captions}

Figure 1. Schematic illustration of doxorubicin (DOX) loaded copper sulfide doped periodic mesoporous organosilicas (DOX-CuS@PMOs) as tri-stimuli-responsive biodegradable theranostics for photothermally enhanced chemotherapy. The three stimuli are glutathione (GSH), $\mathrm{pH}$, and laser irradiation.

Figure 2. TEM images of (a) PMOs and (b) CuS@PMOs. Inset of (a): The photograph of PMOs aqueous solution. Inset of (b): The photograph of CuS@PMOs aqueous solution. (c) UV-vis spectra of POMs and CuS@PMOs. (d) HAADF-STEM image and EDX element mappings (Si, $\mathrm{O}, \mathrm{S}$, and $\mathrm{Cu}$, respectively) of CuS@PMOs. (e) Zeta potentials of PMOs, CuS@PMOs, and DOX-CuS@PMOs. (f) The size distribution of CuS@PMOs and DOX-CuS@PMOs. (g) FT-IR spectra of CuS@PMOs and DOX-CuS@PMOs.

Figure 3. DOX release profiles from DOX-CuS@PMOs at different GSH concentrations and pH values $(\mathrm{a}, \mathrm{b})$ without, and (c, d) with NIR laser irradiation. $* \mathrm{P}<0.05, * * \mathrm{P}<0.01, * * * \mathrm{P}<0.001$.

Figure 4. (a) Confocal images of U87MG cells incubated with fresh medium, free DOX, DOXCuS@PMOs, and DOX-CuS@PMOs with laser irradiation for 30 min (relative DOX concentration: $16 \mu \mathrm{g} / \mathrm{mL}$ ). Scale bar: $10 \mu \mathrm{m}$. (b) Flow cytometry analyses of U87MG cells incubated with different concentrations of DOX-CuS@PMOs for 30 min with laser irradiation. (c) The corresponding mean fluorescence intensity (MFI) of U87MG cells incubated with 
different concentrations of DOX-CuS@PMOs for 30 min without or with laser irradiation. (d) ICP analysis of $\mathrm{Cu}$ uptake by U87MG cells incubated with CuS@PMOs for 30 min without or with laser irradiation by comparison with the cells incubated with fresh medium. $* \mathrm{P}<0.05$.

Figure 5. TEM images of U87MG cells incubated with (a) fresh medium, (b) CuS @PMOs, and $\mathrm{CuS} @$ PMOs with laser irradiation for $30 \mathrm{~min}$ at (c) low and (d) high magnification.

Figure 6. (a) Relative viabilities of U87MG cells incubated with CuS@PMOs with or without $808 \mathrm{~nm}$ laser irradiation for $30 \mathrm{~min}$. (b) Relative viabilities of U87MG cells treated with free DOX, DOX-CuS@PMOs with or without irradiation by $808 \mathrm{~nm}$ laser for $30 \mathrm{~min}$. (c) Fluorescence images of Calcein AM/PI stained U87MG cells incubated with fresh medium, DOX-CuS@PMOs with or without $808 \mathrm{~nm}$ laser irradiation for $30 \mathrm{~min}$, followed by $24 \mathrm{~h}$ incubation with fresh medium (relative DOX concentration: $4 \mu \mathrm{g} / \mathrm{mL}$ ). Scale bar: $100 \mu \mathrm{m}$. ***P $<0.001$.

Figure 7. (a) The fluorescence images of DOX after injection of free DOX and DOX-CuS@PMOs with or without laser irradiation. (b) DOX fluorescence signal in the three groups ( $n=3)$. (c) DOX fluorescence signal at timepoint of 210 min after injection in the three groups. TEM images of sections from tumors injected with DOX-CuS@PMOs (d) with and (e) without irradiation of 808 nm laser. ${ }^{* * *} \mathrm{P}<0.001$.

Figure 8. (a) Photothermal imaging of U87MG tumor-bearing mice injected with PBS and DOXCuS@PMOs upon exposure to 30 min laser irradiation. (b) Tumor growth curves, (c) relative change of 
body weight, and (d) mice survival rate curves of different groups subjected to various treatments. (e) $\mathrm{H} \& \mathrm{E}$ staining of tumors in the five groups. ${ }^{*} \mathrm{P}<0.05,{ }^{* * *} \mathrm{P}<0.001$. 


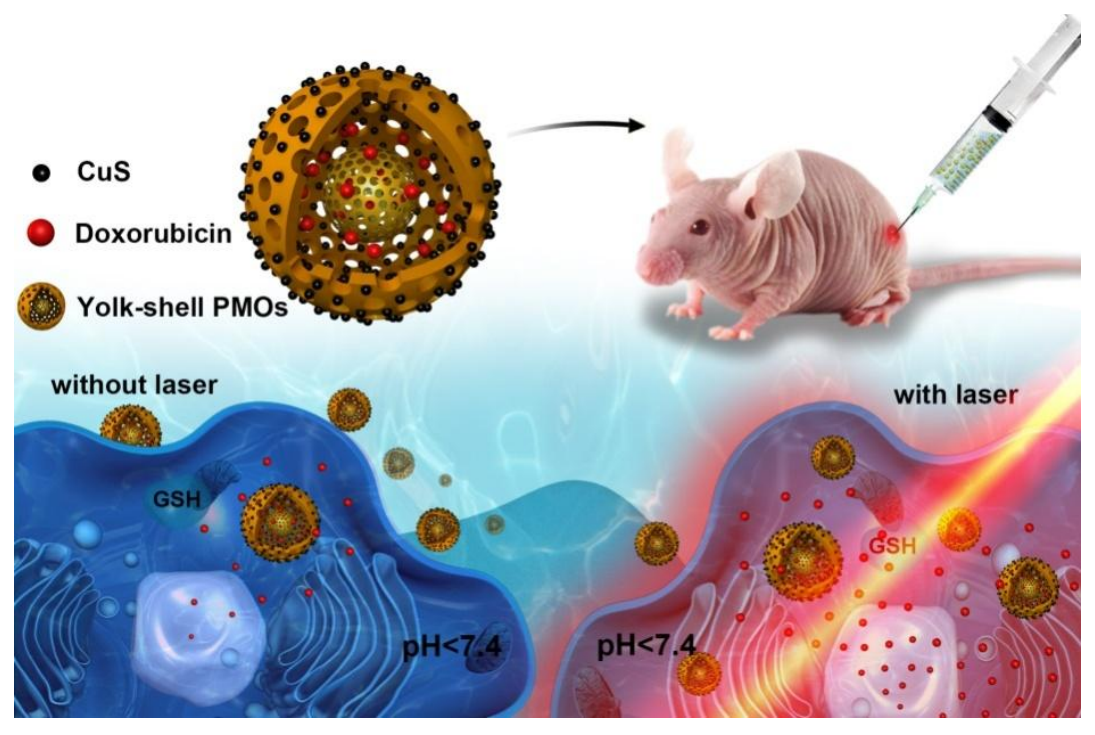

Figure 1 

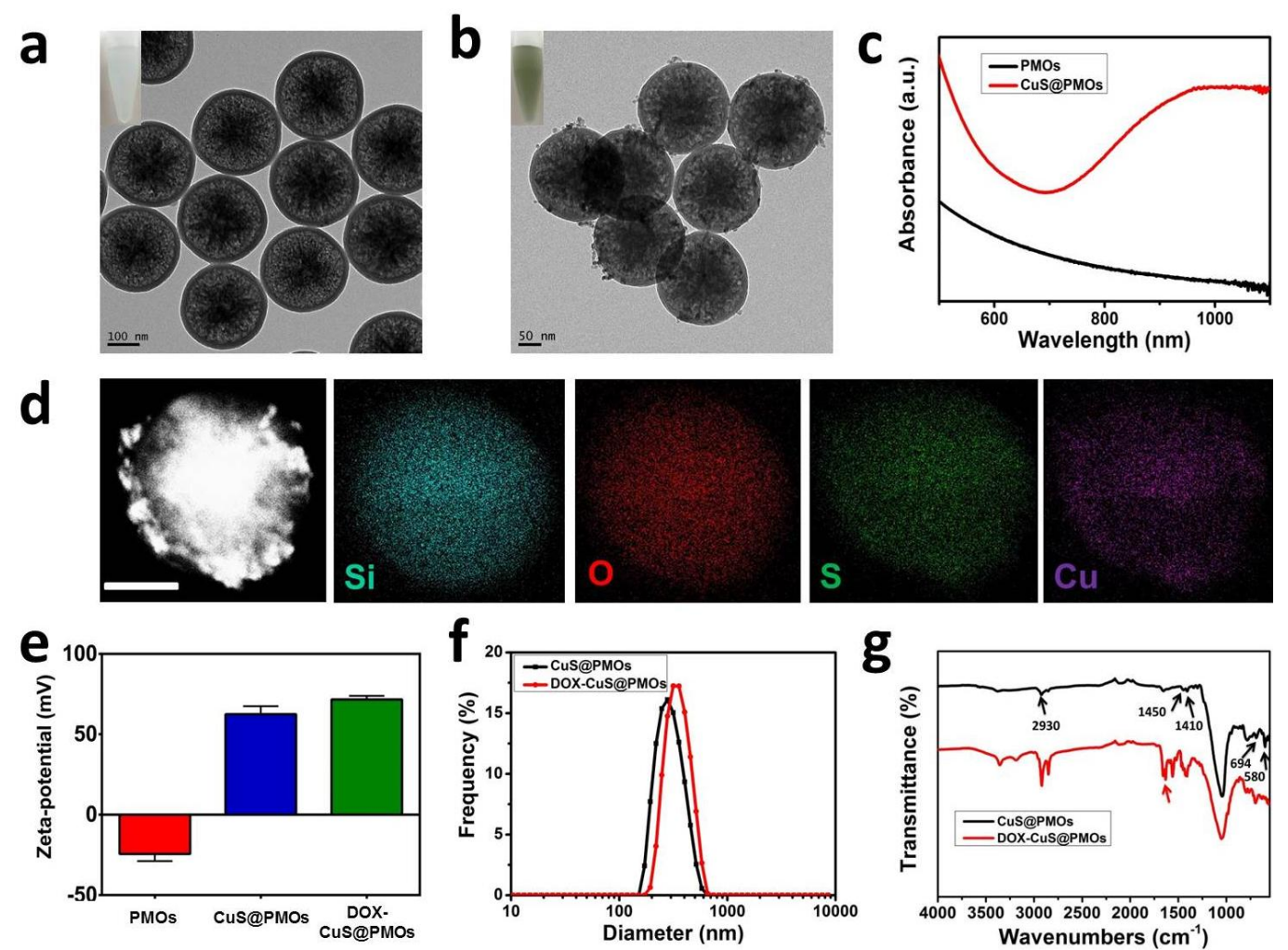

Figure 2 

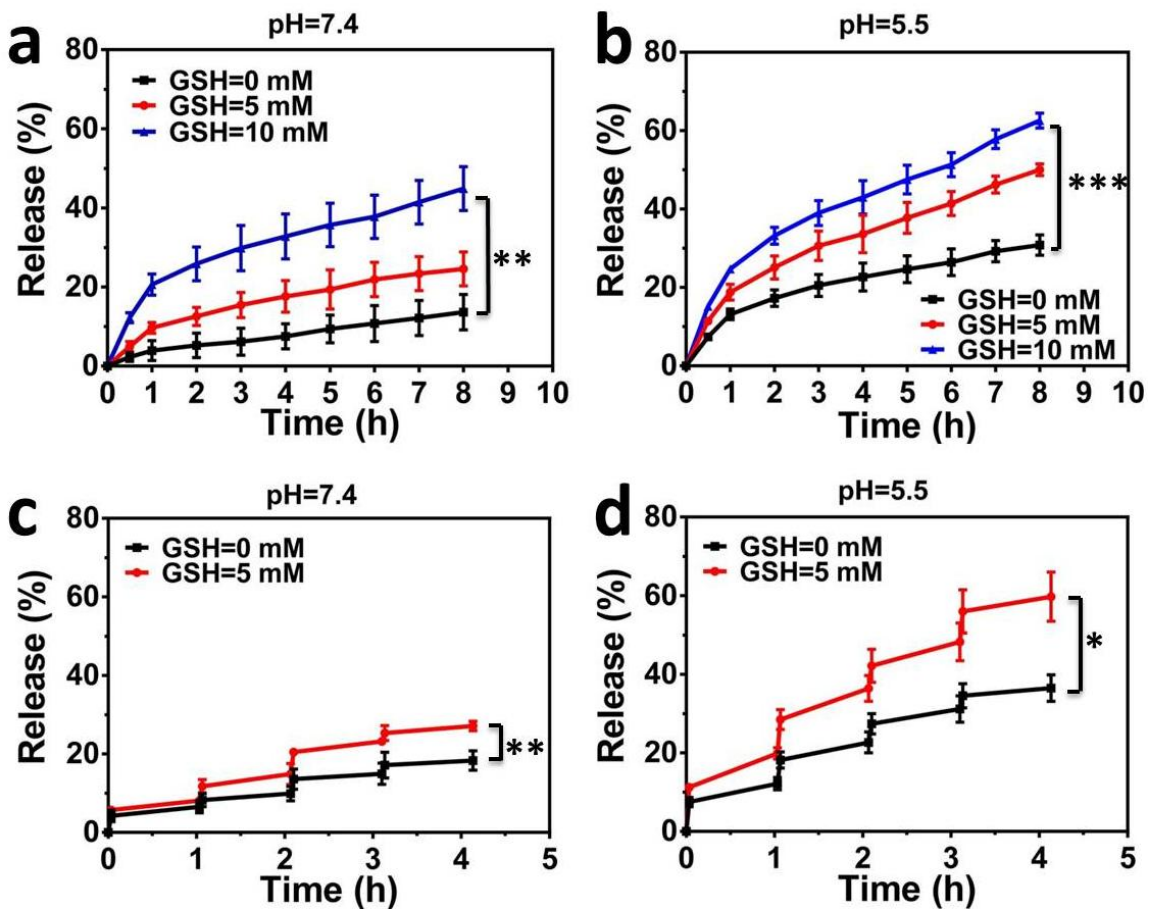

Figure 3 


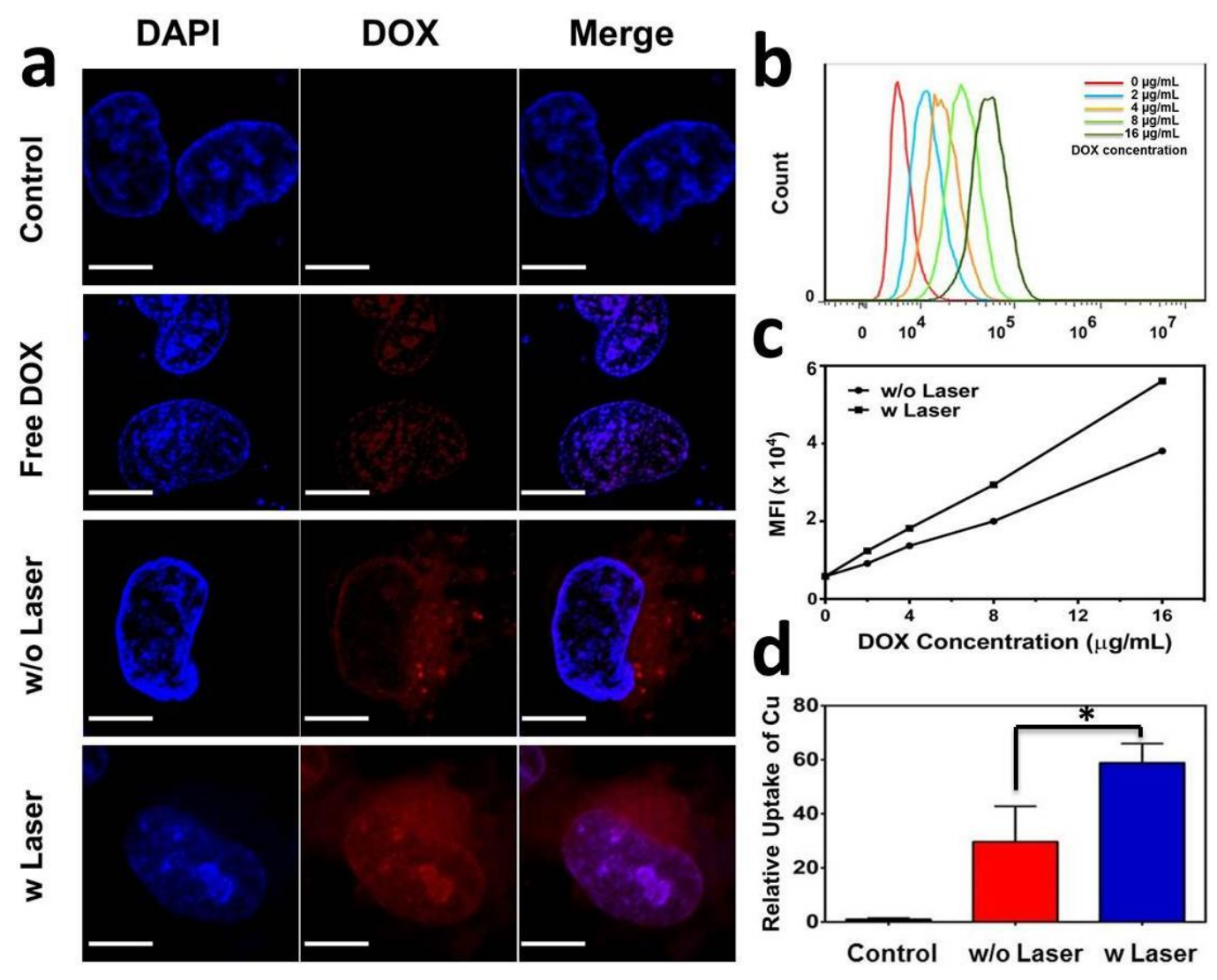

Figure 4 


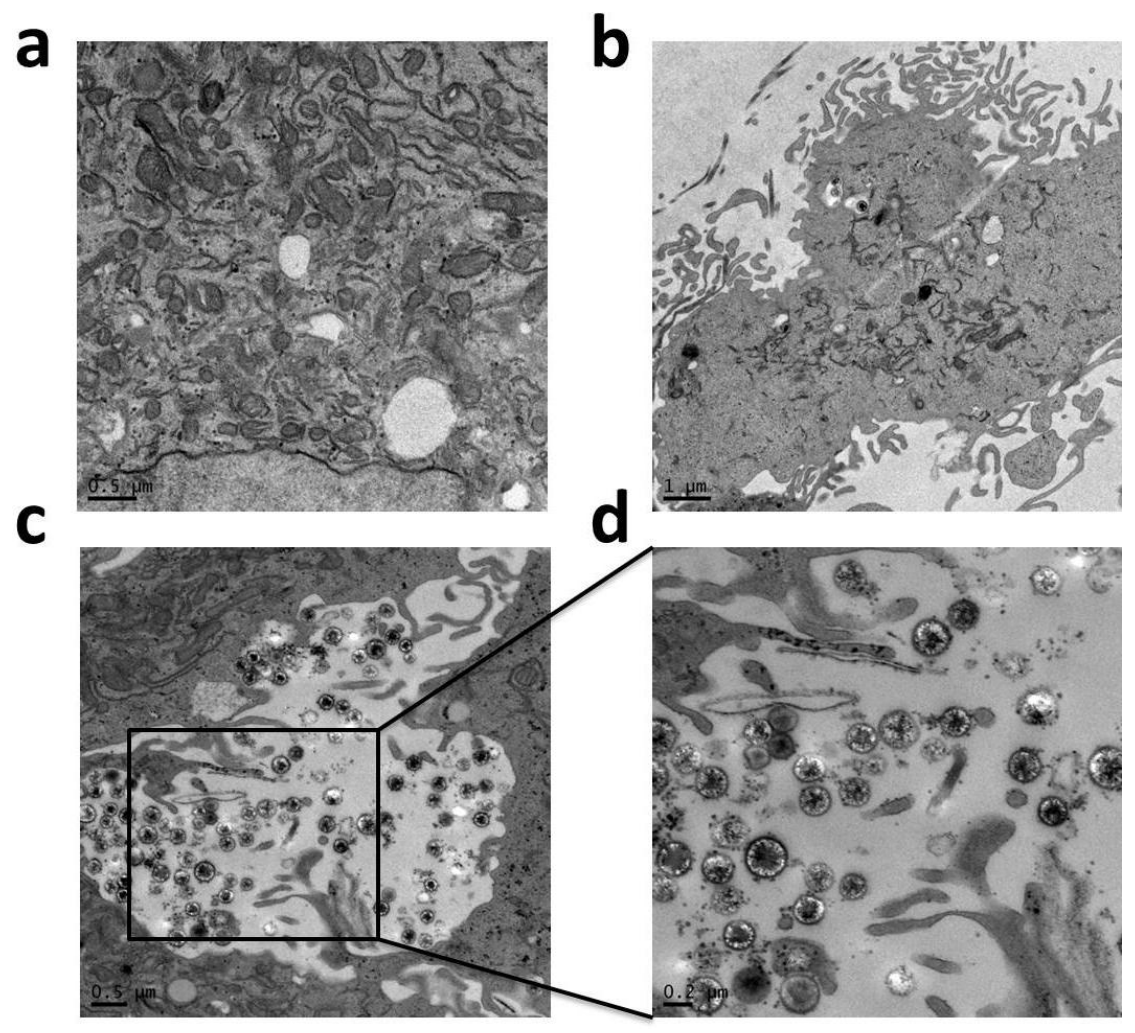

Figure 5 

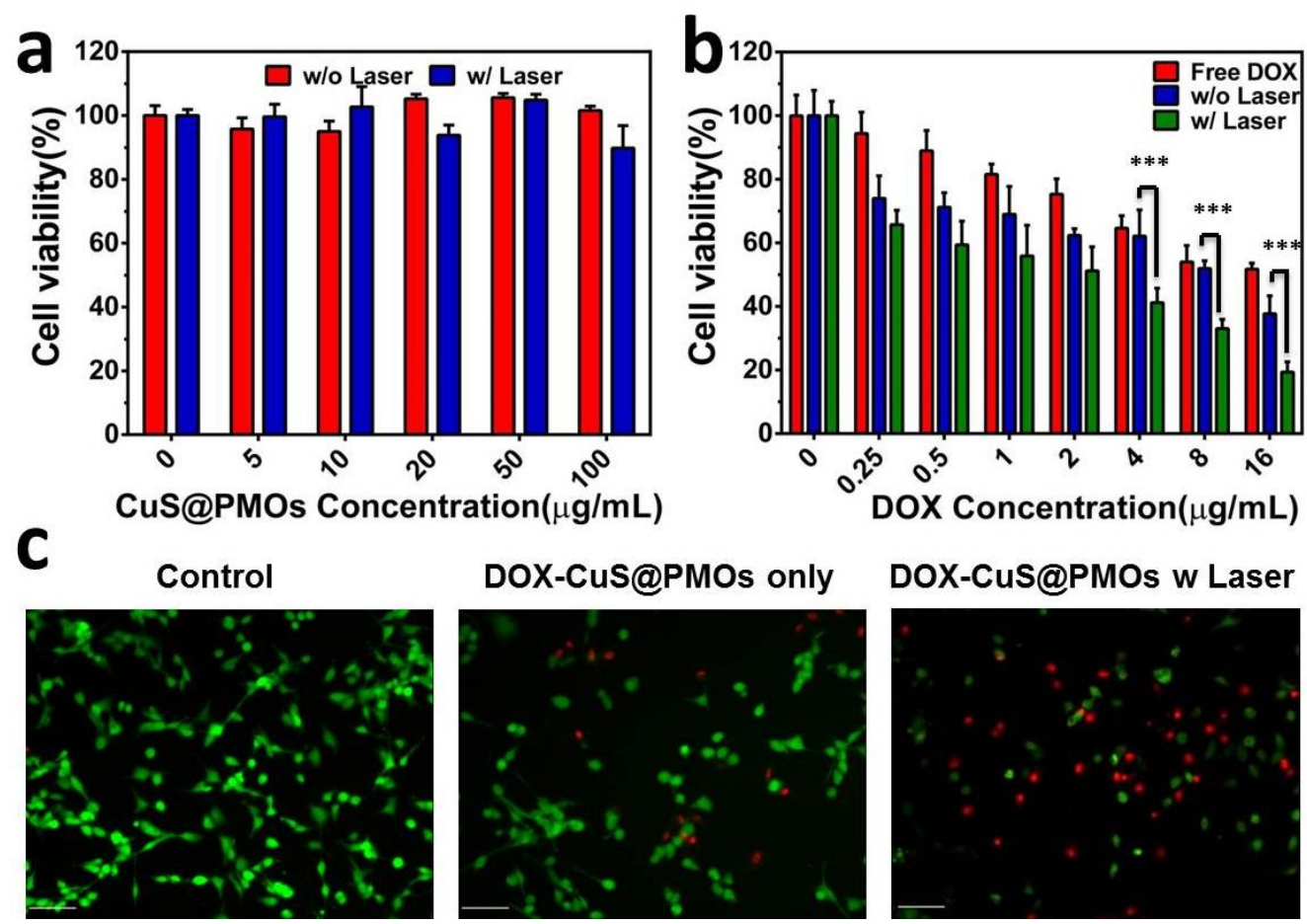

DOX-CuS@PMOs only
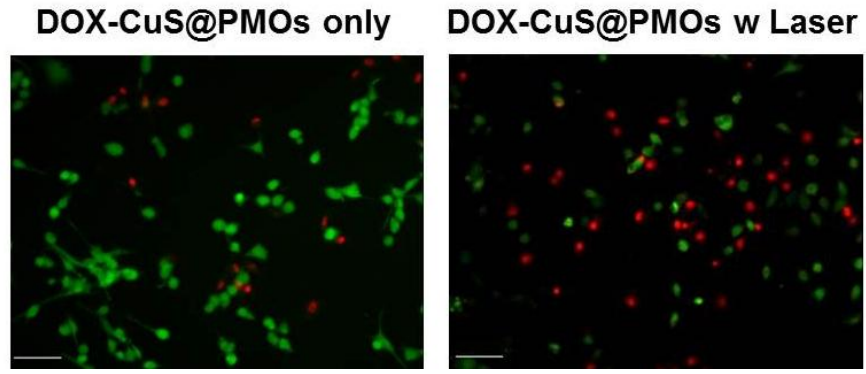

Figure 6 

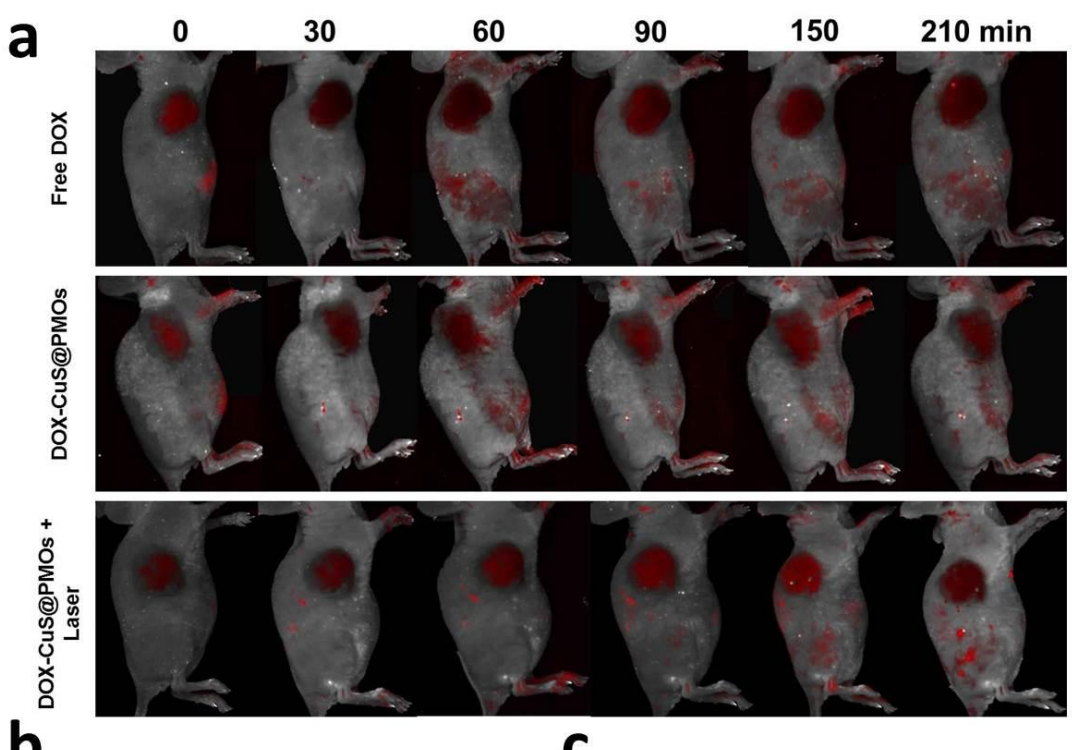

b

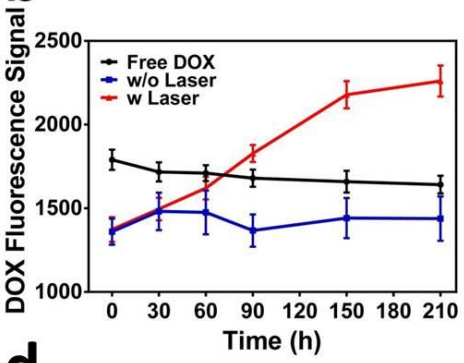

d

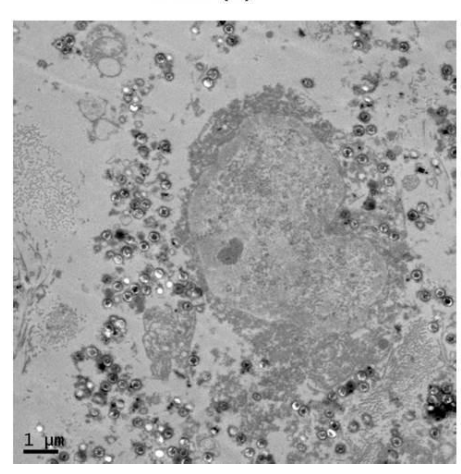

C

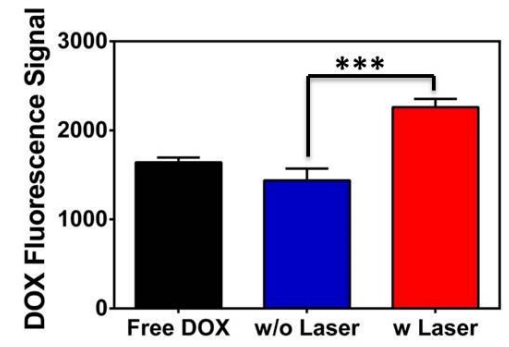

e

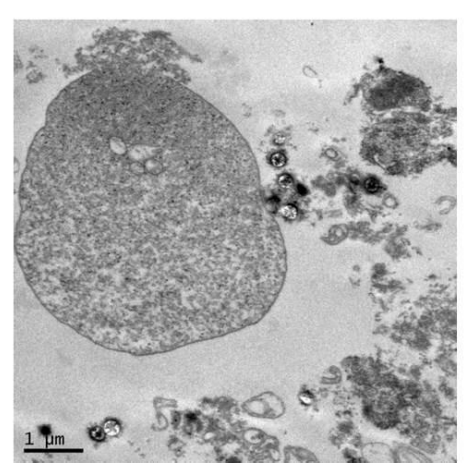

Figure 7 

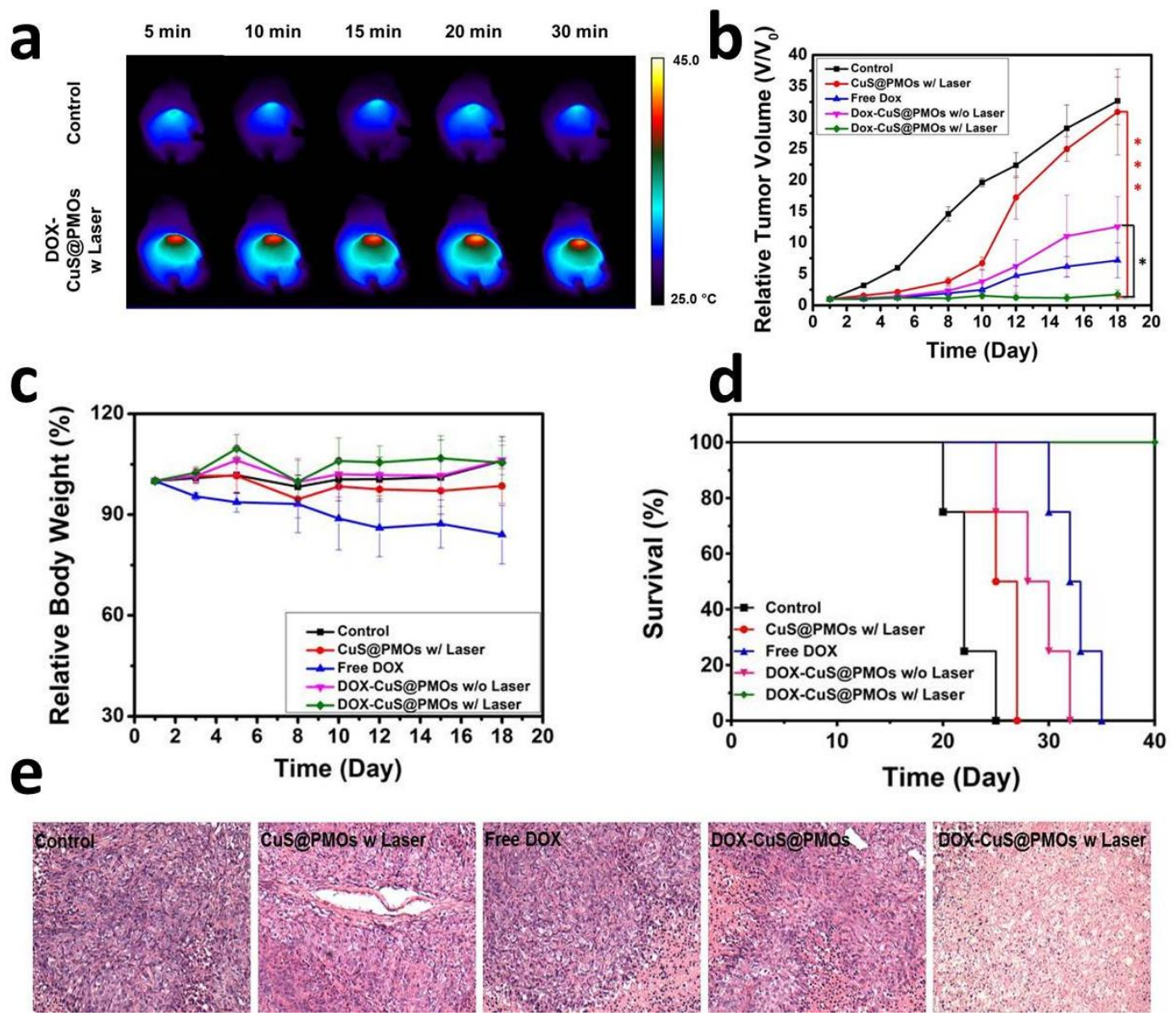

Figure 8 